\title{
CD34 Expression in Noncancerous Liver Tissue Predicts Multicentric Recurrence of Hepatocellular Carcinoma
}

\author{
Naoko Tsuji ${ }^{a}$ Shingo Ishiguro ${ }^{b}$ Yo Sasakic $^{c}$ Masatoshi Kudo $^{d}$ \\ ${ }^{a}$ Department of Gastroenterology, Sakai Hospital, Kinki University Faculty of Medicine, Sakai, Departments of \\ b Pathology and 'Surgery, Osaka Medical Center for Cancer and Cardiovascular Diseases, Osaka, and \\ ${ }^{\mathrm{d}}$ Department of Gastroenterology and Hepatology, Kinki University School of Medicine, Osakasayama, Japan
}

\section{Key Words}

Hepatocellular carcinoma · CD34 expression · Labeling index

\begin{abstract}
Background: Metachronous multicentric recurrence of hepatocellular carcinoma (HCC) is a common cause of morbidity and mortality following curative surgical resection. Clinical and laboratory predictors of these processes can markedly aid in managing these patients. Capillarization of hepatic sinusoids is also a well-known phenomenon in many liver diseases, especially in neoplastic liver diseases. Here, we investigated the clinical features, fibrosis scores and distribution of CD34 in noncancerous hepatic tissues of postresection patients with and without multicentric recurrence. Methods: Eighteen patients with multicentric recurrence of HCC diagnosed by histological examination of repeated hepatectomy specimens and $72 \mathrm{HCC}$ patients with more than 5-year disease-free survival postresection participated in the study. We compared the clinicopathological features of these two groups. We examined noncancerous hepatic tissues for iron deposition by Prussian blue staining and computed the CD34-labeling index (LI) through immunohistochemistry using anti-CD34 antibody. Results: CD34-LI was significantly higher in the multicentric recurrence group $(p<0.001)$ and staging scores of fibrosis were also significantly higher in the recurrence group
\end{abstract}

$(p=0.035)$. A high histological activity grade $(p=0.057)$ and a high alanine aminotransferase level $(p=0.060)$ were also associated with recurrence. There were no significant differences between the two groups in age, sex, hepatitis B virus surface antigen and anti-hepatitis $C$ virus antibody levels, or grade of iron deposition. On multivariate analysis, high CD34-LI was the only independent risk factor $(p=0.001)$ for metachronous multicentric recurrence. Conclusion: CD34 expression in the capillaries and sinusoids of noncancerous hepatic tissue is a risk factor for multicentric recurrence of HCC. Histologic assessment of hepatic tissue with CD34 immunohistochemistry might be useful for the prognostic evaluation of HCC patients after surgery.

๑) 2013 S. Karger AG, Basel

\section{Introduction}

The incidence of intrahepatic recurrence after curative resection of hepatocellular carcinoma (HCC) is very high [1], and many HCC recurrences develop in a multicentric fashion. Effective predictors of multicentric recurrence must be clarified to manage patients after curative hepatectomy. There have been many investigations of cancerous tissues of HCC to predict intrahepatic recurrence, especially intrahepatic metastasis $[2,3]$. To predict multicentric recurrence, we have to investigate noncancerous

\section{KARGER}

E-Mail karger@karger.com

www.karger.com/ddi (c) 2013 S. Karger AG, Basel

0257-2753/13/0316-0467\$38.00/0
Masatoshi Kudo, MD

Department of Gastroenterology and Hepatology, Kinki University School of Medicine 337-2 Ohno-Higashi

Osakasayama, Osaka 589-8511 (Japan)

E-Mail m-kudo@med.kindai.ac.jp 

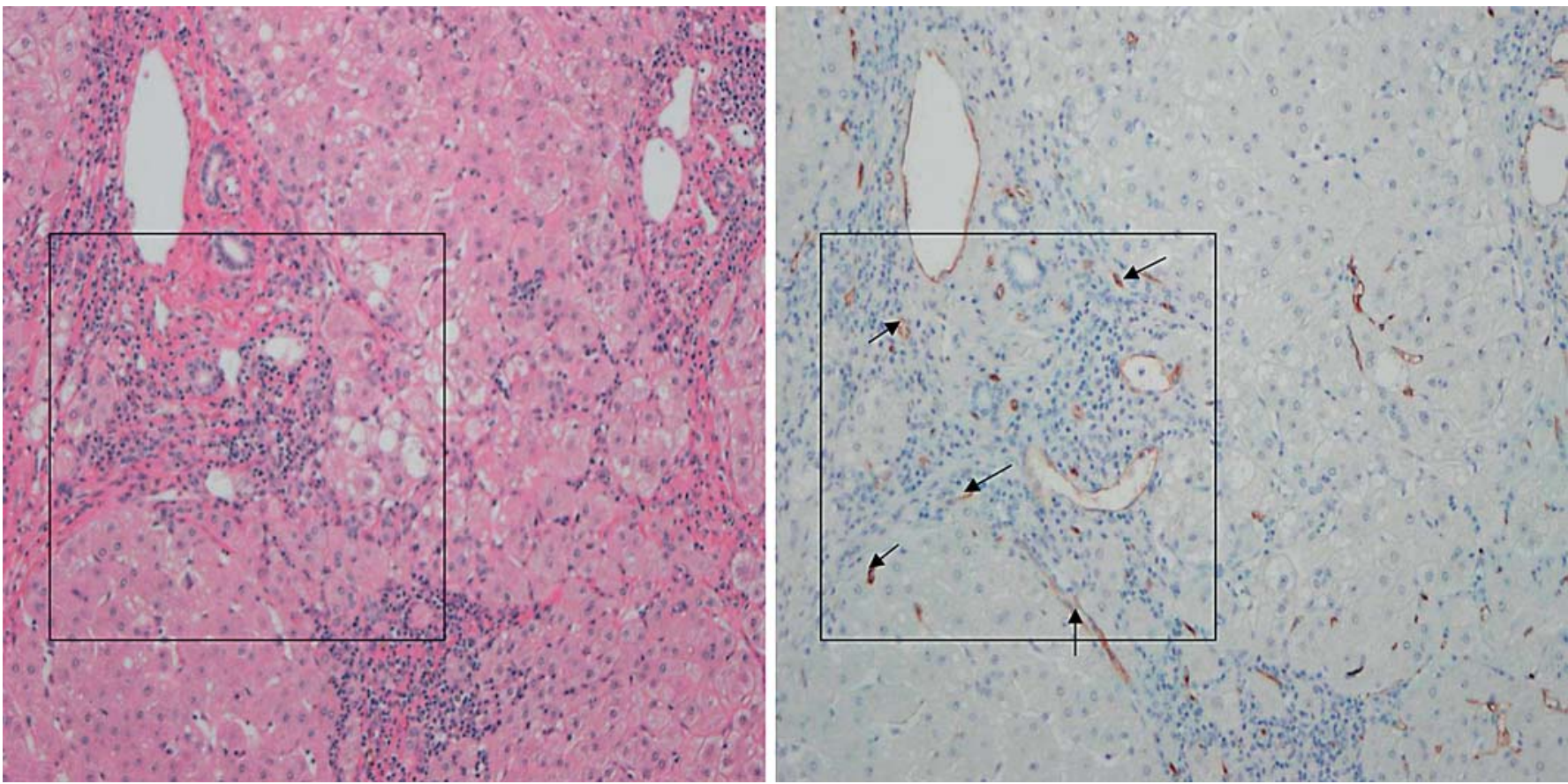

Fig. 1. CD34 immunostaining of noncancerous hepatic tissue. CD34-LI is the average number of CD34-positive capillaries and sinusoids in 10 portal areas. Original magnification $\times 200$.

liver tissue. Multicentric recurrence of HCC is thought to be associated with underlying liver diseases. Angiogenesis and sinusoidal remodeling are closely related to liver inflammation and fibrosis. Normal sinusoidal endothelial cells do not express CD34; however, they show an altered phenotype in chronic liver inflammation to express CD34. In this study, we investigated the noncancerous liver tissue of multicentric recurrence patients and nonrecurrence patients with $\mathrm{CD} 34$ immunostaining to clarify the difference in background livers of those patients.

\section{Material and Methods}

Between 1990 and 1999, 469 HCC patients underwent curative hepatic resection at Osaka Medical Center for Cancer and Cardiovascular Diseases. Metachronous multicentric recurrence of HCC was diagnosed by histological examination of repeated hepatectomy specimens. For the accurate evaluation of a noncancerous background liver, cases in which resected liver specimens were very small or showed degenerative change due to transarterial chemoembolization before surgery were excluded. The definition of metachronous multicentric recurrence was according to the histological criteria of the Liver Cancer Study Group of Japan on the multicentric occurrence of HCC, i.e. recurrent tumors are early HCCs maintaining the existing liver structure, or well-differentiated HCCs found at the edge of moderately or poorly differentiated cancer tissues [4].
Suspected multicentric recurrence patients diagnosed based on clinical images, i.e. CT scan, ultrasonography or needle biopsies of a new nodule after surgery, were also excluded. Eighteen patients met the strict criteria and 72 patients with a more than 5-year disease-free survival were included in our study.

Clinicopathological comparison was made between the 18 patients in the metachronous multicentric recurrence group and the 72 in the nonrecurrence group regarding age, sex, hepatitis B virus surface antigen, anti-hepatitis $\mathrm{C}$ virus (HCV) antibodies and background liver tissue. Paraffin-embedded, noncancerous liver tissue sections were stained with hematoxylin and eosin, and Perls' Prussian blue. The grade of necro-inflammatory activity and stage of fibrosis were classified according to the New Inuyama scoring system for chronic hepatitis [5]. The necro-inflammatory activity and the fibrosis stage were as follows: A0, no necro-inflammatory reaction; A1, mild; A2, moderate, and A3, severe; F0, no fibrosis; F1, fibrous portal expansion; F2, bridging fibrosis; F3, bridging fibrosis with lobular distortion, and F4, cirrhosis. The iron contents in the noncancerous liver tissue were graded according to Seale et al. [6], i.e.: grade 0, absent - iron granules absent/barely discernible at $\times 400$; grade 1 , scarce - barely discernible at $\times 250$ but easily discernible at $\times 400$; grade 2 , mild - discrete granules resolved at $\times 100$; grade 3 , moderate - discrete granules resolved at $\times 25$, and grade 4 , severe - massive iron granules visible at $\times 10$ or with the naked eye.

For immunohistochemistry, anti-CD34 monoclonal mouse antibody (diluted 1:50; QBEnd 10, Dako, Glostrup, Denmark) was used. The avidin-biotin-peroxidase complex immunohistochemistry method (Vectastatin Elite ABC Kit, Vector Laboratories Inc., Burlingame, Calif., USA) was used. CD34-positive capillaries and 
sinusoids of noncancerous liver tissue in 10 portal areas under a high-power field (200× magnifications) were counted, and the average number was defined as the CD34 labeling index (LI; fig. 1).

\section{Statistical Analysis}

Frequencies of various characteristics were compared between the groups with and without multicentric recurrence. Statistical analysis was performed using the $\chi^{2}$ test for categorical data and Mann-Whitney U test for continuous data. A multivariate analysis of risk factors for multicentric recurrence was performed using a logistic regression method. IBM SPSS 17.0 software was used and significance was accepted at $\mathrm{p}<0.05$.

\section{Results}

CD34 immunoreactivity was observed in peribiliary capillaries and periportal and perilobular sinusoids, but no reactivity was shown in parenchymal cells (fig. 1).

\section{Univariate Analysis of Risk Factors Predicting \\ Multicentric Recurrence}

Clinicopathological characteristics with and without metachronous multicentric recurrences are shown in table 1. There were no significant differences in age, sex, hepatitis B virus surface antigen and anti-HCV antibodies between the two groups. The serum alanine aminotransferase (ALT) level $(\mathrm{p}=0.060)$ and a high histological activity grade $(p=0.057)$ showed tendencies to associate with recurrence. Staging scores of fibrosis were significantly higher in the recurrence group $(\mathrm{p}=$ 0.035 ) and CD34-LI was also significantly higher in the multicentric recurrence group $(\mathrm{p}<0.001)$. A high deposition of iron was negatively related to recurrence $(\mathrm{p}=0.086)$.

\section{Multivariate Analysis of Risk Factors Predicting \\ Multicentric Recurrence}

Selected factors that were significant on univariate analysis, including the histological stage of fibrosis and CD34-LI, and other relevant factors, including the serum ALT level, histological grade of activity and grade of iron deposition, were included in the model. CD34-LI and the serum ALT level were converted into categorical data. High CD34-LI (OR, 34.06; 95\% CI, 6.39-181.47; p < 0.001 ) was independently associated with metachronous multicentric recurrence based on multivariate analysis, and a high stage of fibrosis showed a tendency to associate with recurrence $(\mathrm{OR}, 5.62 ; 95 \% \mathrm{CI}, 0.98-31.95 ; \mathrm{p}=$ 0.05 ; table 2).

CD34 Expression Predicts Multicentric Recurrence of HCC
Table 1. Results of univariate analysis between patients with and without metachronous multicentric recurrence

\begin{tabular}{lccr}
\hline & $\begin{array}{l}\text { Recurrence } \\
(+) \\
(\mathrm{n}=18)\end{array}$ & $\begin{array}{l}\text { Recurrence } \\
(-) \\
(\mathrm{n}=72)\end{array}$ & p value \\
\hline Age, years & $59.6 \pm 9.0$ & $59.0 \pm 9.0$ & 0.806 \\
Male sex & $14(78)$ & $58(79)$ & 0.913 \\
ALT level, IU/1 & $97.6 \pm 57.8$ & $75.4 \pm 58.0$ & 0.060 \\
HBsAg positive & $4(22)$ & $13(17)$ & 0.686 \\
HCV-Ab positive & $14(78)$ & $45(59)$ & 0.345 \\
Grade of activity: A2/3 & $17(94)$ & $56(74)$ & 0.057 \\
Stage of fibrosis: F3/4 & $15(83)$ & $40(57)$ & 0.035 \\
Iron deposition: grade 3/4 & $1(6)$ & $17(24)$ & 0.086 \\
CD34-LI & $45.2 \pm 23.1$ & $14.3 \pm 7.9$ & $<0.001$ \\
\hline
\end{tabular}

Values are either mean \pm SD or number with percentage in parentheses. Statistical analysis was performed using the $\chi^{2}$ test for categorical and Mann-Whitney U test for continuous data.

Table 2. Results of multivariate analysis of the risk factors for metachronous multicentric recurrence

\begin{tabular}{lrrl}
\hline & p value & OR & \multicolumn{1}{l}{$95 \%$ CI } \\
\hline ALT >100 IU/1 & 0.18 & 2.89 & $0.61-13.71$ \\
Grade of activity: A2/3 & 0.39 & 3.25 & $0.21-50.07$ \\
Stage of fibrosis: F3/4 & 0.05 & 5.62 & $0.98-31.95$ \\
Iron deposition: grades 3/4 & 0.27 & 0.23 & $0.17-3.16$ \\
CD34-LI $>25$ & $<0.01$ & 34.06 & $6.39-181.47$ \\
\hline
\end{tabular}

\section{Discussion}

The intrahepatic recurrence rate of HCC after curative surgical resection remained very high. Long-term followup data after surgery showed that the recurrence rate of $\mathrm{HCV}$-positive HCC patients was $80 \%$, and that of HBVpositive HCC patients was 59\% [7]. There are two different types of recurrence: intrahepatic metastasis and multicentric recurrence. In Japan, 45-60\% of recurrence after surgical resection was reported to be multicentric. Kumada et al. [8] reported that $50.9 \%$ of recurrence after ethanol infection therapy was multicentric. However in China, Li et al. [9] reported that the rate of multicentric recurrence after surgical resection was $30 \%$. We adopted histopathological criteria to strictly differentiate whether the recurrent HCC was intrahepatic metastasis or multicentric recurrence, and several clonal analyses of both primary HCC and recurrent HCC were reported for a more accu- 
rate diagnosis [10-12]. However, according to a nationwide survey, repeat hepatectomy has been performed in $1.6 \%$ of all patients [13]. Therefore, in many clinical situations, the mode of recurrence was diagnosed based on clinical data such as vascular invasion of primary HCC, the period of recurrence, size and site of the recurrent tumor, and hemodynamics of the recurrent tumor assessed by dynamic CT or contrast-enhanced ultrasonography. The risk factors for synchronous and metachronous multicentric occurrence of HCC were reported to be: male gender, HCV positive, aged, low platelet count, high grading and staging scores, high ALT activity, and high concentration of type 4 collagen [14-16]. Tarao et al. [17] reported the increased DNA synthesis activity of hepatocytes in the residual liver of multicentric recurrence patients using an in vitro BrdU assay of biopsied specimens, and, recently, a few studies have investigated gene expression in noncancerous liver tissue to predict risk factors of multicentric recurrence $[18,19]$. In our study, the multicentric recurrence group also showed a significantly higher stage of fibrosis and tendency toward a higher ALT activity and grade of activity on univariate analysis; however, regarding the HCV status, there were no significant differences between the two groups. Many HCV positive patients in our study showed recurrence after curative hepatectomy, and many of them had suspected multicentric recurrence according to the clinical findings, but did not undergo repeat hepatectomy because of the functional reserve of the liver or selection of another therapy.

In our study, CD34 expression in noncancerous liver tissue was a significant risk factor of multicentric recurrence on uni- and multivariate analyses. CD34 antigen is expressed in hematopoietic stem cells and endothelial cells. In normal liver tissue, CD34 expression is restricted to portal vascular structures. It is also detected in oval cells in rat liver [20], but we identified no immunoreactivity in hepatocytes or oval cell-like cells in this study. The hepatic sinusoidal endothelial cells have numerous holes (fenestrae) and lack a basement membrane. They do not contain molecules characteristically found in the vascular endothelium, such as CD34 and GMP-140. However, in chronic liver disease, intrahepatic angiogenesis and sinusoidal remodeling occurs, characterized by the deposition of a basement membrane, loss of sinusoidal endothelial fenestrae, and by the expression of CD34, which is commonly referred to as the 'capillarization' of sinusoids [21, 22]. Angiogenesis of chronic liver disease evaluated by CD34 positivity is correlated with the degree of fibrosis [23], and strong staining was reported in HCC and hepatocellular adenoma [24-27]. Ohmori et al. [28, 29] reported that high expression of CD34-positive sinusoidal endothelial cells was a risk factor for developing HCC in HCV and HBV chronic hepatitis patients using liver biopsy specimens. Angiogenesis occurs under conditions associated with tissue damage, wound healing and remodeling. Angiogenesis also occurs in tumors. High CD34-LI in noncancerous liver tissue seems to be the result of severe tissue damage and inflammation. To prevent multicentric recurrence, antiinflammatory therapy is essential, as long-term interferon therapy reduced the carcinogenesis rate of HCV patients [30]. Are angiogenesis and sinusoidal remodeling new targets for preventing carcinogenesis? Sorafenib is a multikinase inhibitor compound approved for liver cancer and it inhibits tumor angiogenesis. In animal models, sorafenib also inhibits both matrix restructuring and vascular remodeling that accompanies chronic liver disease [31]. It may be a candidate for a new therapy to treat liver fibrosis and prevent carcinogenesis, but further investigation is needed before clinical application to humans.

Liver iron overload was reported as a risk factor for HCC. Liver iron accumulation causes oxidative stress and leads to liver cell DNA mutations, and the development of HCC. Several studies reported that liver iron overload contributed to the development of HCC in patients with viral $\mathrm{C}$ hepatitis $[32,33]$. In our study, iron deposition in noncancerous liver tissue had a negative impact on multicentric recurrence. Nahon et al. [34] reported that liver iron overload is associated with a higher risk of HCC in patients with alcoholic but not HCV-related cirrhosis, and Sorrentino et al. [35] reported that iron deposition was associated with NASH-related cirrhosis. In our study, the nonrecurrence group included patients whose etiologies of hepatitis were nonviral.

This study has some limitations. We selected patients with multicentric recurrence diagnosed by repeat hepatectomy; therefore, the number of studied patients was small, and the histological types of second primary HCC were limited to the early HCC type or well-differentiated type. Therefore, our multicentric recurrence group had some biases.

In conclusion, CD34 expression in the capillaries and sinusoids of noncancerous hepatic tissue is a risk factor for the multicentric recurrence of HCC. Histologic assessment of hepatic tissue with CD34 immunohistochemistry is a simple and straightforward method, and might be useful for the prognostic evaluation of HCC patients after surgery.

\section{Disclosure Statement}

The authors have no conflicts of interest to declare. 


\section{References}

1 Kubo S, Takemura S, Sakata C, Urata Y, Uenishi T: Adjuvant therapy after curative resection for hepatocellular carcinoma associated with hepatitis virus. Liver Cancer 2013;2:4046.

2 Kim YK: Prediction of HCC recurrence by CT and MRI. Liver Cancer 2013;2:181.

3 Burak KW: Prediction of hepatocellular carcinoma recurrence by molecular biomarkers. Liver Cancer 2013;2:185.

4 Japan Liver Cancer Study Group: General Rules for the Clinical and Patholoical Study of Primary Liver Cancer, English ed 3. Tokyo, Kanehara 2010, p 54.

5 Ichida F, Tsuji T, Omata M, Ichida T, Inoue K, Kamimura T, Yamada G: New Inuyama classification; new criteria for histological assessment of chronic hepatitis. Int Hepatol Commun 1996;6:112-119.

6 Seale J, Leggett BA, Crawford DHG, Powell LW: Iron storage disease; in MacSween RNM, Burt AD, Portmann BC, Ishak KG, Scheuer PJ, Anthony PP (eds): Pathology of the Liver, ed 4. Edinburgh, Churchill Livingstone, 2002, p 260.

$\checkmark 7$ Sasaki Y, Yamada T, Tanaka H, Ohigashi H, Eguchi H, Yano M, Ishikawa O, Imaoka S: Risk of recurrence in a long-term follow-up after surgery in 417 patients with hepatitis Bor hepatitis C-related hepatocellular carcinoma. Ann Surg 2006;244:771-780.

$>8$ Kumada T, Nakano S, Takeda I, Sugiyama K, Osada T, Kiriyama S, Sone Y, Toyoda H, Shimada S, Takahashi M, et al: Patterns of recurrence after initial treatment in patients with small hepatocellular carcinoma. Hepatology 1997;25:87-92.

$>9$ Li Q, Wang J, Juzi JT, Sun Y, Zheng H, Cui Y, Li H, Hao X: Clonality analysis for multicentric origin and intrahepatic metastasis in recurrent and primary hepatocellular carcinoma. J Gastrointest Surg 2008;12:1540-1547.

10 Morimoto O, Nagano H, Sakon M, Fujiwara Y, Yamada T, Nakagawa H, Miyamoto A, Kondo M, Arai I, Yamamoto T, et al: Diagnosis of intrahepatic metastasis and multicentric carcinogenesis by microsatellite loss of heterozygosity in patients with multiple and recurrent hepatocellular carcinomas. J Hepatol 2003;39:215-221.

-11 Tsuda H, Oda T, Sakamoto M, Hirohashi S: Different pattern of chromosomal allele loss in multiple hepatocellular carcinomas as evidence of their multifocal origin. Cancer Res 1992;52:1504-1509.

12 Nakashima O, Kojiro M: Recurrence of hepatocellular carcinoma: multicentric occurrence or intrahepatic metastasis? A viewpoint in terms of pathology. J Hepatobiliary Pancreat Surg 2001;8:404-409.

$>13$ Arii S, Teramoto K, Kawamura T, Okamoto H, Kaido T, Mori A, Imamura M: Characteristics of recurrent hepatocellular carcinoma in Japan and our surgical experience. J Hepatobiliary Pancreat Surg 2001;8:397-403.
14 Kubo S, Yamamoto T, Ikebe T, Shuto T, Hirohashi $\mathrm{K}$, Tanaka $\mathrm{H}$, Tsukamoto $\mathrm{T}$, Wakasa K, Kinoshita H: Relationship between multicentric occurrence of hepatocellular carcinoma and histology of noncancerous hepatic tissue in patients with chronic hepatitis C. Jpn J Cancer Res 1999;90:1076-1080.

15 Ikeda K, Arase Y, Kobayashi M, Saitoh S, Someya T, Hosaka T, Suzuki Y, Suzuki F, Tsubota A, Akuta N, et al: Significance of multicentric cancer recurrence after potentially curative ablation of hepatocellular carcinoma: a longterm cohort study of 892 patients with viral cirrhosis. J Gastroenterol 2003;38: 865-876.

16 Tomimaru Y, Sasaki Y, Yamada T, Eguchi H, Ohigashi H, Ishikawa O, Imaoka S: Fibrosis in non-cancerous tissue is the unique prognostic factor for primary hepatocellular carcinoma without hepatitis B or C viral infection. World J Surg 2006;30:1729-1735.

17 Tarao K, Hoshino H, Shimizu A, Ohkawa S, Nakamura Y, Harada M, Ito Y, Tamai S, Akaike M, Sugimasa Y, et al: Role of increased DNA synthesis activity of hepatocytes in multicentric hepatocarcinogenesis in residual liver of hepatectomized cirrhotic patients with hepatocellular carcinoma. Jpn J Cancer Res 1994;85:1040-1044.

18 Okamoto M, Utsunomiya T, Wakiyama S, Hashimoto M, Fukuzawa K, Ezaki T, Hanai T, Inoue $\mathrm{H}$, Mori $\mathrm{M}$ : Specific gene-expression profiles of noncancerous liver tissue predict the risk for multicentric occurrence of hepatocellular carcinoma in hepatitis $\mathrm{C}$ virus-positive patients. Ann Surg Oncol 2006;13:947954.

19 Hoshida Y, Villanueva A, Kobayashi M, Peix J, Chiang DY, Camargo A, Gupta S, Moore J, Wrobel MJ, Lerner J, et al: Gene expression in fixed tissues and outcome in hepatocellular carcinoma. New Engl J Med 2008;359:19952004.

20 Omori N, Omori M, Evarts RP, Teramoto T, Miller MJ, Hoang TN, Thorgeirsson SS: Partial cloning of rat CD34 cDNA and expression during stem cell-dependent liver regeneration in the adult rat. Hepatology 1997;26:720-727.

21 Schaffner F, Poper H: Capillarization of hepatic sinusoids in man. Gastroenterology 1963;44:239-242.

22 Couvelard A, Scoazec JY, Feldmann G: Expression of cell-cell and cell-matrix adhesion proteins by sinusoidal endothelial cells in the normal and cirrhotic human liver. Am J Pathol 1993;143:738-752.

23 Amarapurkar AD, Amarapurkar DN, Vibhav S, Patel ND: Angiogenesis in chronic liver disease. Ann Hepatol 2007;6:170-173.

24 Ruck P, Xiao JC, Kaiserling E: Immunoreactivity of sinusoids in hepatocellular carcinoma: an immunohistochemical study using lectin UEA-1 and antibodies against endothelial markers, including CD34. Arch Pathol Lab Med 1995;119:173-178.
25 Kimura H, Nakajima T, Kagawa K, Deguchi T, Kakusui M, Katagishi T, Okanoue T, Kashima $\mathrm{K}$, Ashihara T: Angiogenesis in hepatocellular carcinoma as evaluated by CD34 immunohistochemistry. Liver 1998;18:14-19.

$>26$ Maeda T, Adachi E, Kajiyama K, Takenaka K, Honda H, Sugimachi K, Tsuneyoshi M: CD34 expression in endothelial cells of small hepatocellular carcinoma: its correlation with tumour progression and angiographic findings. J Gastroenterol Hepatol 1995;10:650-654.

27 Cui S, Hano H, Sakata A, Harada T, Liu T, Takai S, Ushigome S: Enhanced CD34 expression of sinusoid-like vascular endothelial cells in hepatocellular carcinoma. Pathol Int 1996; 46:751-756.

28 Ohmori S, Shiraki K, Sugimoto K, Sakai T, Fujikawa K, Wagayama H, Takase K, Nakano T: High expression of CD34-positive sinusoidal endothelial cells is a risk factor for hepatocellular carcinoma in patients with $\mathrm{HCV}$-associated chronic liver diseases. Hum Pathol 2001;32:1363-1370.

29 Ohmori S, Shiraki K, Sugimoto K, Yamanaka Y, Yamaguchi Y, Saitou Y, Fujikawa K, Murata K, Nakano T: Expression of CD34-positive sinusoidal endothelial cells in patients with $\mathrm{HBV}$-associated chronic liver diseases. Int J Mol Med 2004;14:179-184.

30 Ikeda K, Saitoh S, Kobayashi M, Suzuki Y, Suzuki F, Tsubota A, Arase Y, Murashima N, Chayama K, Kumada H: Long-term interferon therapy for 1 year or longer reduces the hepatocellular carcinogenesis rate in patients with liver cirrhosis caused by hepatitis $\mathrm{C}$ virus: a pilot study. J Gastroenterol Hepatol 2001;16:406-415.

31 Thabut D, Routray C, Lomberk G, Shergill U, Glaser K, Huebert R, Patel L, Masyuk T, Blechacz $\mathrm{B}$, Vercnocke A, et al: Complementary vascular and matrix regulatory pathways underlie the beneficial mechanism of action of sorafenib in liver fibrosis. Hepatology 2011;54:573-585.

32 Tanaka H, Fujita N, Sugimoto R, Urawa N, Horiike S, Kobayashi Y, Iwasa M, Ma N, Kawanishi S, Watanabe S, et al: Hepatic oxidative DNA damage is associated with increased risk for hepatocellular carcinoma in chronic hepatitis C. Br J Cancer 2008;98:580-586.

33 Chapoutot C, Esslimani M, Joomaye Z, Ramos J, Perney P, Laurent C, Fabbro-Peray P, Larrey D, Domergue J, Blanc F: Liver iron excess in patients with hepatocellular carcinoma developed on viral C cirrhosis. Gut 2000;46:711-714.

34 Nahon P, Sutton A, Rufat P, Ziol M, Thabut G, Schischmanoff PO, Vidaud D, Charnaux $\mathrm{N}$, Couvert P, Ganne-Carrie N, et al: Liver iron, HFE gene mutations, and hepatocellular carcinoma occurrence in patients with cirrhosis. Gastroenterology 2008;134:102-110.

35 Sorrentino P, D’Angelo S, Ferbo U, Micheli P, Bracigliano A, Vecchione R: Liver iron excess in patients with hepatocellular carcinoma developed on non-alcoholic steato-hepatitis. J Hepatol 2009;50:351-357. 